\title{
Algoritmo de Balanceamento Inter-Núcleos para Redes Ópticas Elásticas com Multiplexação por Divisão Espacial
}

\author{
Jurandir Lacerda Jr ${ }^{1,2}$, Alexandre Fontinele ${ }^{3}$, Iallen Santos ${ }^{3,4}$, Erico Leão ${ }^{1}$, \\ Divanilson R. Campelo ${ }^{3}$, José Suruagy Monteiro ${ }^{3}$, André Soares ${ }^{1}$ \\ ${ }^{1}$ Departamento de Computação - Universidade Federal do Piauí (UFPI) \\ Teresina-PI \\ ${ }^{2}$ Instituto Federal de Educação, Ciência e Tecnologia do Piauí (IFPI) \\ Corrente-PI \\ ${ }^{3}$ Centro de Informática (CIn) - Universidade Federal de Pernambuco (UFPE) \\ Recife-PE \\ ${ }^{4}$ Instituto Federal de Educação, Ciência e Tecnologia do Piauí (IFPI) \\ Piripiri-PI \\ jurandir.cavalcantedifpi.edu.br
}

\begin{abstract}
Recently, elastic optical networks with spatial division multiplexing (SDM) have been considered a promising solution to compose the Internet infrastructure. This paper proposes ABNE - "Algoritmo de Balanceamento de Núcleo e Espectro para Redes SDM”. The proposal makes a balanced allocation of core and spectrum to minimize the impact of crosstalk in the optical circuits. The proposal is evaluated by computer simulation. It is efficient in terms of blocking probability and energy efficiency compared to other proposals. ABNE produced a reduction in the blocking probability a minimum of $44 \%$ in the NSFNet topology and $33 \%$ in the EON topology.
\end{abstract}

Resumo. Recentemente, as redes ópticas elásticas com multiplexação por divisão espacial (SDM) apresentam-se como solução promissora para compor a infraestrutura da Internet. Este trabalho propõe o Algoritmo de Balanceamento de Núcleo e Espectro para Redes SDM (ABNE). Tal proposta busca uma alocação balanceada de núcleo e espectro, de modo a minimizar o impacto do crosstalk nos circuitos ópticos. A proposta é avaliada via simulação computacional e mostra-se eficiente em termos de probabilidade de bloqueio e eficiência energética quando comparada com outras soluções da literatura. $O$ ABNE alcança uma redução na probabilidade de bloqueio de pelo menos $44 \%$ na topologia NSFNet e $33 \%$ na topologia EON.

\section{Introdução}

A cada dia, novas aplicações que usam a Internet são propostas. Soluções de computação na nuvem, telemedicina, ou stream de vídeo em ultra definição (4K) como a Netflix, Amazon Prime Video e Disney Plus demandam alta capacidade da infraestrutura de comunicação. Para suprir esta e outras demandas, a comunicação óptica deve ser aprimorada para dar suporte a esta crescente demanda de tráfego. 
Neste sentido, as redes ópticas elásticas (Elastic Optical Network - EON) [Jinno et al. 2009] surgem como uma solução para compor a infraestrutura da Internet. Em uma rede óptica elástica, o espectro óptico é dividido em pequenas seções ópticas denominadas slots de frequência. Este tipo de divisão oferece maior eficiência na alocação de recursos quando comparada aos modelos de grade fixa. [Chatterjee et al. 2015]. Recentemente, a literatura tem estudado um modelo de multiplexação conhecido como multiplexação por divisão espacial (Space Division Multiplexing - SDM) [Klinkowski and Zalewski 2019, Oliveira and Fonseca 2019]. Neste modelo, o espectro óptico é dividido espacialmente, através de núcleos distintos dentro de uma única fibra. Assim, redes EON-SDM podem lidar com maiores volumes de tráfego.

Para a alocação de um circuito óptico em uma rede EON-SDM deve-se considerar o problema de roteamento, alocação de núcleo e espectro (Routing, Spectrum and Core Assignment - RSCA) [Lobato et al. 2019]. O problema RSCA consiste em: i) definir a rota que o circuito irá percorrer do nó de origem até o nó de destino; ii) Selecionar qual núcleo será usado para o transporte dos dados; e iii) quais slots serão alocados para o circuito. Durante a transmissão, o sinal óptico sofre interferências que podem inviabilizar a comunicação entre o transmissor e receptor. Estudar interferências como o crosstalk torna a modelagem mais realista. O problema RSCA pode ser dividido em dois subproblemas: i) o subproblema de roteamento e ii) o subproblema de alocação de núcleo e espectro (Spectrum and Core Assignment - SCA) [Lobato et al. 2019].

O escopo deste artigo é o desenvolvimento de soluções para o subproblema SCA para uma rede EON-SDM. Neste sentido, o Algoritmo de Balanceamento de Núcleo e Espectro (ABNE) é proposto. O ABNE realiza um balanceamento na escolha de núcleo e de espectro para mitigar o efeito do crosstalk na rede. Este balanceamento viabiliza redução de probabilidade de bloqueio e aumento na eficiência energética da rede.

O restante deste artigo está organizado da seguinte forma. A Seção 2 apresenta as redes ópticas elásticas com multiplexação SDM e suas características. A Seção 3 exibe um levantamento do estado da arte sobre redes EON-SDM. Na Seção 4 é apresentado o algoritmo proposto neste artigo. A Seção 5 apresenta a avaliação de desempenho da proposta. E, por fim, as conclusões e trabalhos futuros são apresentados na Seção 6.

\section{Redes Ópticas Elásticas com Multiplexação SDM}

Em uma rede óptica elástica com multiplexação por divisão espacial (EON-SDM), cada fibra óptica possui múltiplos núcleos, sendo cada núcleo dividido em pequenos canais de frequência denominados slots de frequência. A Figura 1 ilustra uma rede multi-núcleos.
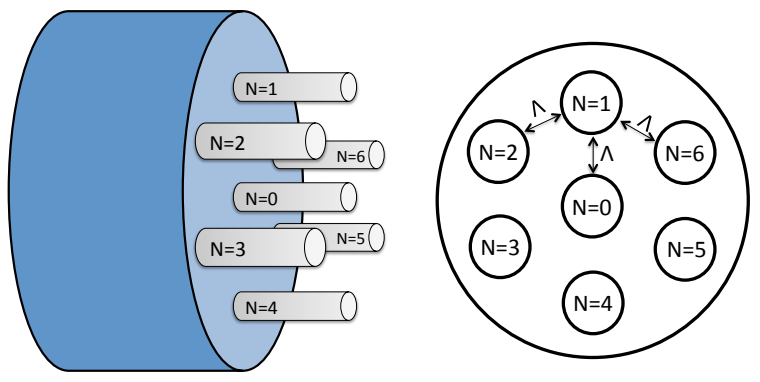

Figura 1. Exemplo de fibras multi-núcleos. Fibra com sete núcleos. 
A Figura 1 mostra a divisão de uma fibra em sete núcleos usando SDM. Cada um dos núcleos possui seu próprio conjunto de slots, estando separados por uma distância $\Lambda$. Redes multi-núcleos podem conter um número variado de núcleos. Neste trabalho, considera-se redes com sete núcleos. Tal quantidade está de acordo com outros trabalhos da literatura [Oliveira and Fonseca 2018] [Ehsani Moghaddam et al. 2019].

Cada requisição para criação de um novo circuito óptico contém as informações de origem, destino e taxa de transmissão. A partir destas informações, o algoritmo RSCA seleciona um núcleo e um conjunto de slots para suprir essa demanda. A quantidade de slots varia de acordo com a demanda e do formato de modulação usado. Considerando a existência de recurso disponível na rede, o requisitante terá à sua disposição um circuito óptico dedicado. Caso não exista disponibilidade de recurso, a requisição é negada. Este evento é conhecido como bloqueio de requisição.

Para a alocação de recursos, é necessário definir qual conjunto de slots o circuito óptico vai usar. Esta definição deve obedecer a duas restrições [Chatterjee et al. 2015]: $i$ ) contiguidade espectral, que exige que todos os slots de um circuito devem ser adjacentes entre si (isto é, estarem em frequências adjacentes); ii) continuidade espectral, que exige que a frequência de cada slot deve ser a mesma em todos os enlaces do circuito.

Diferentes técnicas podem ser utilizadas para a alocação espectral, como os algoritmos first fit, last fit, best fit e random fit [Chatterjee et al. 2015]. O first fit sempre escolhe, quando possível, o conjunto de slots mais próximos do inicio do espectro (menor frequência). O last fit procura alocar o intervalo de slots no final do espectro óptico (maior frequência). No modelo best fit, o intervalo escolhido é aquele com o mesmo tamanho da requisição (quando possível), não importando se está no inicio ou final do espectro. Por fim, o random fit escolhe o conjunto de slots de forma aleatória.

\subsection{Imperfeições da Camada Física}

Naturalmente, ocorre uma degradação da qualidade do sinal óptico durante sua propagação. Isso acontece devido aos efeitos de camada física, que surgem nos enlaces ou em outros dispositivos da rede. Usualmente, a amplificação óptica é realizada pelo amplificador à fibra dopada com ébrio (Erbium Doped Fiber Amplifer - EDFA). Amplificadores EDFA acabam também inserindo o ruído emissão espontânea amplificada (Amplied Spontaneous Emission - ASE) [Yan et al. 2015]. Existem ainda outros efeitos que causam interferência nos circuitos, como a auto-modulação de fase (Self-Phase Modulation - SPM), a modulação de fase cruzada (Cross-Phase Modulation - XPM) e a mistura de quatro ondas (Four-Wave Mixing - FWM). Os efeitos ASE, SPM, XPM e FWM são considerados neste trabalho, por estarem em consonância com o que a literatura trata.

Em uma rede óptica elástica, os efeitos de camada física impactam na qualidade de transmissão (Quality of Transmission - QoT) do circuito. Uma maneira de mensurar os níveis de QoT é através da relação sinal ruído (Signal to Noise Ratio - SNR). A SNR estabelece uma relação entre a densidade espectral de potência do sinal (Power Spectral Density - PSD) do circuito com a PSD dos ruídos que a atingem. A SNR é usada como critério de QoT para este trabalho. Segundo [Johannisson and Agrell 2014, Yan et al. 2015], o cálculo da SNR para um circuito $i$ usando uma rota $r_{i}$ é expresso por:

$$
S N R_{i}=\frac{I}{I_{A S E}+I_{N L I}} .
$$


A variável $I$ é a densidade espectral da potência do sinal (Power Spectral Density - PSD), $I=P_{T R} / \Delta_{f}$, em que $P_{T R}$ é potência de sinal e $\Delta_{f}$ é a largura de banda do circuito. A PSD do ruído ASE é dada por:

$$
I_{A S E}=\sum_{l \in r_{i}} N_{l} I_{A S E},
$$

em que $N_{l}$ é o número de spans do enlace $l$. Um span é um segmento de enlace composto por uma fibra óptica e um equipamento amplificador, necessário para enlaces que cubram longos caminhos [Yan et al. 2015]. O termo $I_{A S E}=\left(G_{A M P}-1\right) F h v$. A variável $F$ é a figura de ruído (Noise Figure - NF) do amplificador. $h$ é a constante de Planck, $v$ é a frequência da luz e $G_{A M P}$ é o ganho do amplificador óptico. A PSD do ruído dos efeitos não lineares (SPM, XPM e FWM) é dada por:

$$
I_{N L I}=\sum_{l \in r_{i}} N_{l} I_{N L I}
$$

em que $I_{N L I}$ é a PSD do ruído NLI em um único span do enlace $l$. A $I_{N L I}$ é expressa pela Equação 4 [Zhao et al. 2015]:

$$
I_{N L I}=\frac{3 \gamma^{2} I^{3}}{2 \pi \alpha\left|\beta_{2}\right|}\left(\operatorname{arcsinh}\left(\frac{\pi^{2}\left|\beta_{2}\right|}{2 \alpha} B_{i}^{2}\right)+\sum_{j} \ln \left[\frac{\left(\Delta_{f_{i j}}+\frac{B_{j}}{2}\right)}{\left(\Delta_{f_{i j}}-\frac{B_{j}}{2}\right)}\right]\right),
$$

em que, $j$ é outro circuito usando o enlace $l, B i$ e $B j$ são, respectivamente, as larguras de bandas para os circuitos $i$ e $j, \Delta_{f_{i j}}$ é o espaçamento da frequência central entre os circuitos $i$ e $j, \gamma$ é o coeficiente não linear da fibra, $\beta_{2}$ é o coeficiente de dispersão de velocidade de grupo e $\alpha$ é a atenuação de potência causada pela fibra.

Além das imperfeições de camada física apresentadas, outra importante característica de uma rede SDM é o crosstalk (XT). O crosstalk inter-núcleos é um fenômeno que ocorre quando a mesma frequência (slot) é utilizada entre núcleos próximos. Quanto menor a distância entre os núcleos, maior o efeito do crosstalk. Em [Lobato et al. 2019] é apresentada uma modelagem para mensurar a interferência causada pelo crosstalk em uma rede SDM. A Equação 5 apresenta o crosstalk para um determinado circuito óptico [Lobato et al. 2019].

$$
X T_{\mu, i}^{(t o t)}=\sum_{l=1}^{N_{i}} X T_{\mu, i}^{(l)}
$$

em que $N_{i}$ é a quantidade de enlaces do circuito e $X T_{\mu, i}^{(l)}$ é o crosstalk de um enlace, dado pela Equação 6.

$$
X T_{\mu, i}=\frac{P_{X T i}}{P_{S i}},
$$

Na Equação 6, $P_{X T i}$ é o valor do crosstalk no enlace $i$ e $P_{S i}$ corresponde a potência do sinal de $i$. $\mathrm{O}$ valor de $P_{X T i}$ pode ser encontrado pela Equação 7 .

$$
P_{X T i}=\sum_{j=1}^{N_{a}}\left(I_{S O i j} \cdot P_{S j} \cdot h \cdot L\right),
$$


em que $N_{a}$ é o número de núcleos adjacentes a $i$. $I_{S O i j}$ é o índice de sobreposição de slots entre os circuitos $i$ e $j$ (Equação 8). $P_{S j}$ é a potência de sinal do circuito adjacente $j$. $L$ é o tamanho do enlace de $i$ e $h$ pode ser calculado pela Equação 9.

$$
I_{S o i j}=\frac{N_{S o i j}}{N_{S j}}
$$

Na Equação 8, $N_{\text {Soij }}$ é a quantidade de slots sobrepostos entre $i$ e $j$. Isto é, quantos dos slots de $i$ possuem slots de mesmo índice alocados em $j$ (desconsiderando banda de guarda). E $N_{S j}$ é a quantidade de slots de $j$.

$$
h=\frac{2 \cdot k^{2} \cdot R}{\beta \cdot \Lambda} .
$$

Na Equação 9, $k$ é o coeficiente de acoplamento, $R$ é o raio de curvatura, $\beta$ constante de propagação e $\Lambda$ é a distância entre núcleos. A Figura 2 apresenta o fluxo do processo de estabelecimento de um novo circuito adotado neste trabalho, considerando os efeitos de camada física.
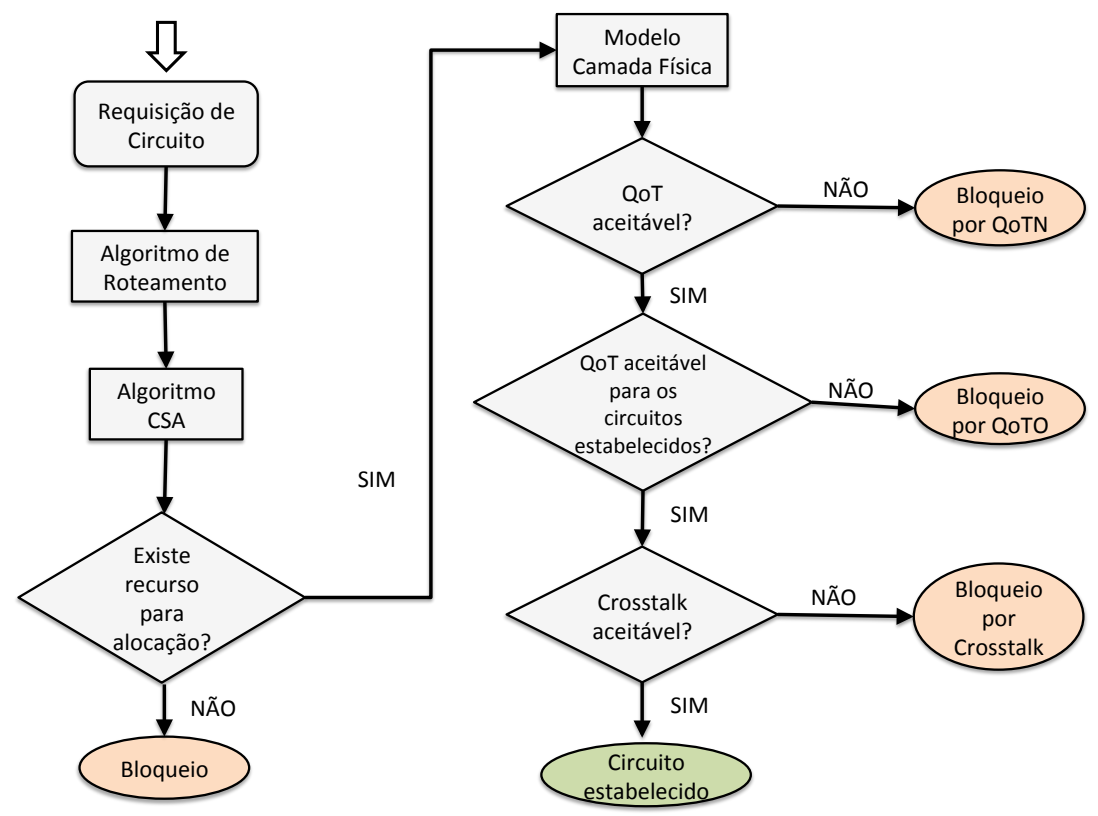

Figura 2. Fluxograma do modelo de alocação de recursos.

A seguir são discutidas as etapas apresentadas na Figura 2. Ao chegar a requisição para o estabelecimento de um novo circuito, o algoritmo RSCA calcula uma rota entre o nó de origem $(o)$ e o nó de destino $(d)$ juntamente com o núcleo e o conjunto de slots a serem usados. Caso não existam recursos livres, a requisição será bloqueada. Existindo disponibilidade de recursos, o modelo de camada física analisa a QoT (através da SNR) e o nível de crosstalk para o circuito candidato. Caso a QoT do circuito candidato não esteja adequada, ocorrerá um bloqueio por ausência de QoT no novo circuito (QoTN). Caso contrário, será analisado o impacto da possível alocação deste circuito nos circuitos já estabelecidos. Se tal impacto afetar algum dos circuitos já estabelecidos de tal forma 
que a sua SNR fique em desacordo com o limiar estabelecido, o novo circuito sofrerá um bloqueio por ausência de QoT para os outros circuitos (QoTO). Por fim, observa-se o crosstalk para a requisição, caso o valor seja aceitável, o circuito é estabelecido.

\section{Trabalhos Relacionados}

As EON-SDM têm se destacado como objeto de estudos nos últimos anos. A maioria dos trabalhos abordam o problema RSCA para este tipo de rede, considerando questões como o crosstalk além de outros problemas de camada física [Rottondi et al. 2018, Ehsani Moghaddam et al. 2019, Klinkowski and Zalewski 2019]. Também são estudados variantes do problema RSCA, que adicionam novos desafios ao problema, como a escolha adaptativa de modulação ou garantia de sobrevivência da rede.

Em [Moura and Fonseca 2018] os autores apresentam uma solução para o problema de roteamento, escolha de núcleo, formato de modulação e alocação de espectro (Routing, Core, Modulation Level, and Spectrum Assignment - RCMLSA) para redes ópticas elásticas SDM. É proposta uma abordagem de representação espectral diferenciada, usando matrizes e algoritmos de reconhecimento de imagens para a identificação de sequencias de slots disponíveis. Tal proposta mostrou-se eficiente, além de reduzir a complexidade computacional para o problema.

Os autores em [Lobato et al. 2019] propõem um algoritmo guloso, ciente de crosstalk, para resolver o problema SCA para redes EON-SDM. Tal algoritmo busca minimizar o impacto do crosstalk tanto nos novos circuitos quanto nos circuitos já estabelecidas. Para isso, o algoritmo busca, no conjunto de soluções, a solução com maior valor de $\triangle X T$. Isto é, o circuito candidato com valor de crosstalk mais distante do limiar de crosstalk estabelecido para a rede.

Os autores em [Oliveira and Fonseca 2019] apresentam o algoritmo Multipath Protection for Multicore network (MPMN). Tal algoritmo foi proposto para garantir sobrevivência em redes ópticas elásticas SDM. Para garantir sobrevivência, o MPMN emprega a estratégia de proteção de caminho. Além disso, a proposta usa roteamento multicaminhos para diminuir o bloqueio gerado pela fragmentação do espectro.

Em [Fujii et al. 2014] é proposto um método dinâmico da classificação de núcleos com base nas larguras de banda necessárias para redes multi-núcleos. O método proposto busca a priorização de determinados núcleos com objetivo de diminuir o crosstalk. Além disso, também é proposto um método de classificação de núcleos com o objetivo de diminuir a fragmentação da rede.

Os autores em [Klinkowski and Zalewski 2019] desenvolveram um algoritmo de aprovisionamento de lightpath ciente de crosstalk para redes EON-SDM. A proposta realiza uma estimativa precisa de crosstalk em um cenário dinâmico, com o uso de múltiplos formatos de modulação. Também foi avaliado o impacto do crosstalk em cenário estático, considerando o pior caso de interferência.

Em todos os trabalhos citados é recorrente o estudo do impacto do crosstalk nas redes EON-SDM. De maneira geral, existem três formas de considerar o crosstalk durante a solução do problema SCA [Lobato et al. 2019]: i) XT-avoid, onde o algoritmo SCA tenta evitar sobreposição de slots em núcleos adjacentes; ii) XT-worst case, que considera sempre o pior cenário de crosstalk; e iii) XT-aware, que usa valores em tempo 
real de crosstalk para a tomada de decisão. Apesar da sua eficiência, algoritmos XT-aware tendem a ter maior complexidade computacional em relação ao XT-avoid e XT-worst case. Buscando resolver o problema SCA de forma eficiente e com baixa complexidade computacional, o algoritmo proposto neste trabalho enquadra-se na classe XT-avoid.

\section{Algoritmo Proposto}

Neste artigo é proposto o Algoritmo de Balanceamento de Núcleo e Espectro para Redes SDM (ABNE). O ABNE busca um balanceamento na escolha de núcleo e espectro para mitigar o efeito do crosstalk na rede. Considerando que o crosstalk afeta slots de mesmo índice em núcleos vizinhos, a ideia é priorizar porções de slots distintos entre os núcleos adjacentes. A Figura 3 apresenta a ideia geral do algoritmo.

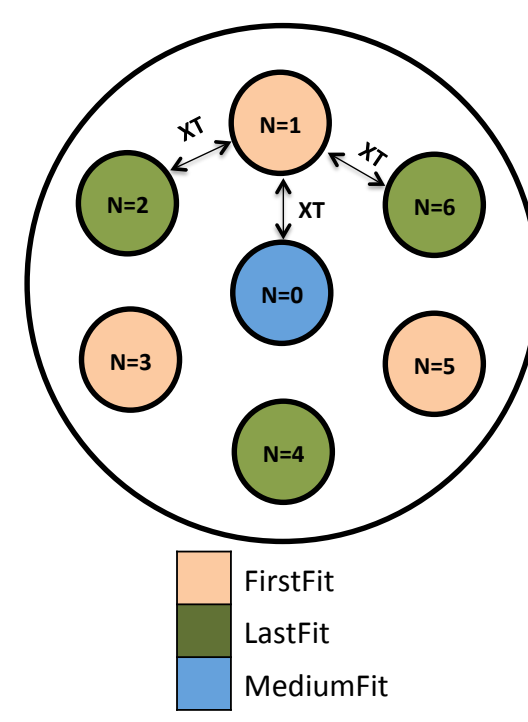

(a)

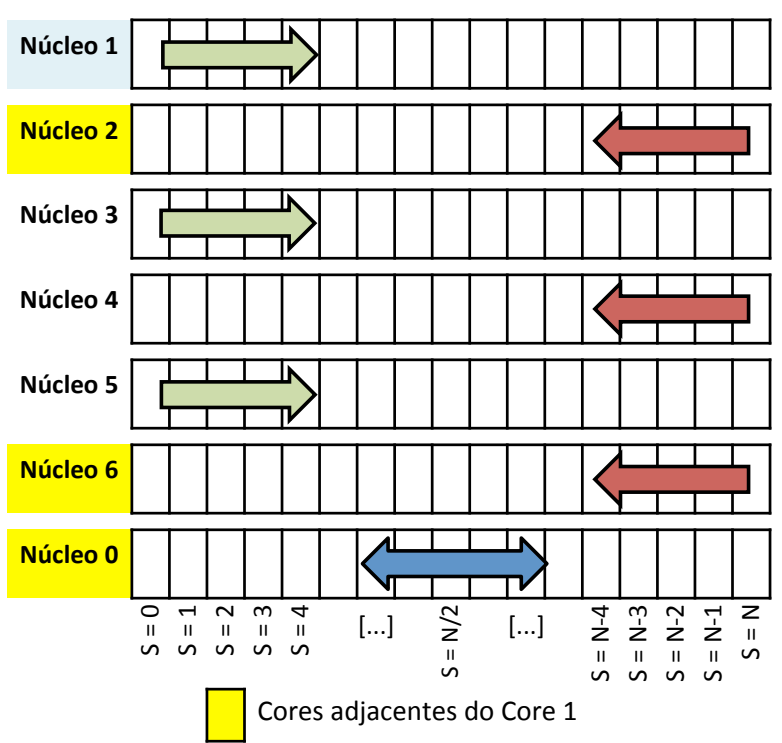

(b)

Figura 3. Algoritmo ABNE.

Na Figura 3 (a) é exemplificado como é a disposição dos núcleos em uma rede SDM de sete núcleos. De maneira geral, o algoritmo ABNE busca ocupar os slots de núcleos vizinhos em sentidos opostos. Tomando como exemplo a Figura 3 (b), o algoritmo ABNE ocupa o núcleo 1 utilizando os slots disponíveis de menor índice (algoritmo first fit). Por outro lado, os slots dos núcleos 2 e 6 (vizinhos do núcleo 1) são alocados do maior para o menor índice (last fit). Vale ressaltar que o núcleo 0 (ver Figura 3) é ao mesmo tempo vizinho de todos os núcleos. Portanto, para o núcleo 0, o algoritmo ABNE aloca os slots partindo do centro para as extremidades. Esse comportamento diferenciado para a alocação no núcleo 0 visa diminuir a sua interferência (crosstalk) em todos os outros núcleos da fibra. Portanto, a estratégia do algoritmo ABNE evita a alocação de slot de mesmo índice em núcleos vizinhos. O Algoritmo 1 apresenta o pseudocódigo do algoritmo ABNE.

O Algoritmo 1 apresenta o pseudocódigo do ABNE. No caso, os núcleos 1, 3 e 5 buscam os slots mais próximos do início do espectro (algoritmo first fit), enquanto os núcleos 2, 4 e 6 priorizam os slots do final do espectro (algoritmo last fit). Já o núcleo 0 (núcleo central), que influencia no crosstalk de todos os outros, prioriza os slots distantes 


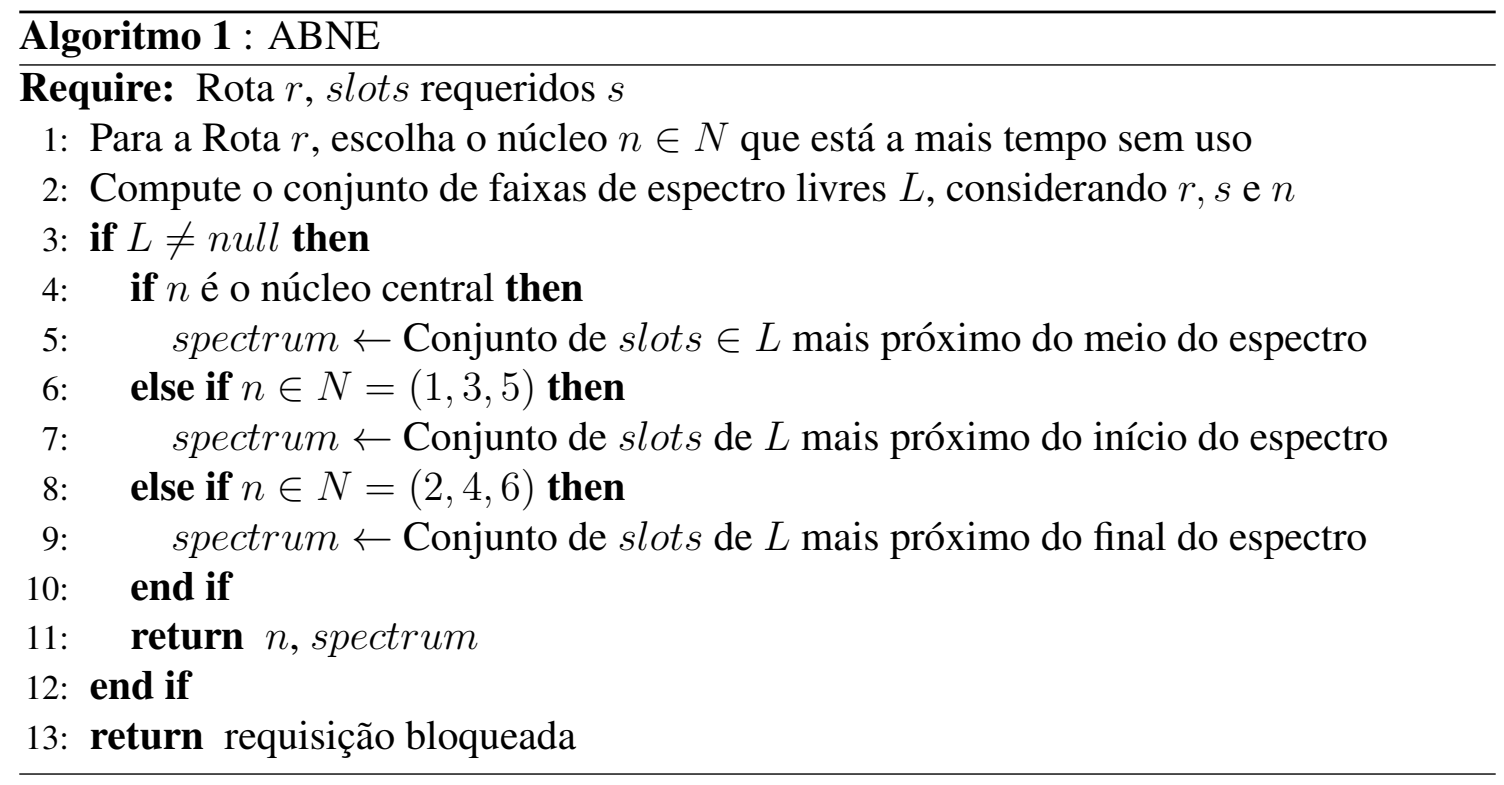

do inicio e do final do espectro concomitantemente. Assim, a alocação espectral é feita visando minimizar o crosstalk na rede.

\section{Avaliação de Desempenho}

Para o estudo de avaliação de desempenho deste trabalho, foi utilizado o simulador SLICE Network Simulator (SNetS) [Fontinele et al. 2017]. Para as simulações foram geradas 100.000 requisições de circuitos em cada simulação. A geração de requisições é um processo de Poisson com taxa média de $\lambda$ e o tempo médio de retenção dos circuitos é distribuído exponencialmente com média $1 / \mu$. A carga de tráfego é distribuída uniformemente entre todos os pares de nós origem e destino. São geradas requisições de 100 , 150, 200, 250, 300, 350 e $400 \mathrm{Gbps}$, seguindo a proporção de chegadas de 7, 6, 5, 4, $3,2,1$, respectivamente. A carga, em Erlangs, pode ser definida por $\rho=\lambda / \mu$. Para cada simulação foram realizadas 10 replicações com diferentes sementes de geração de variável aleatória. Todos os resultados possuem nível de confiança de 95\%. As topologias consideradas nas simulações são a NSFNet e a EON (Figura 4).
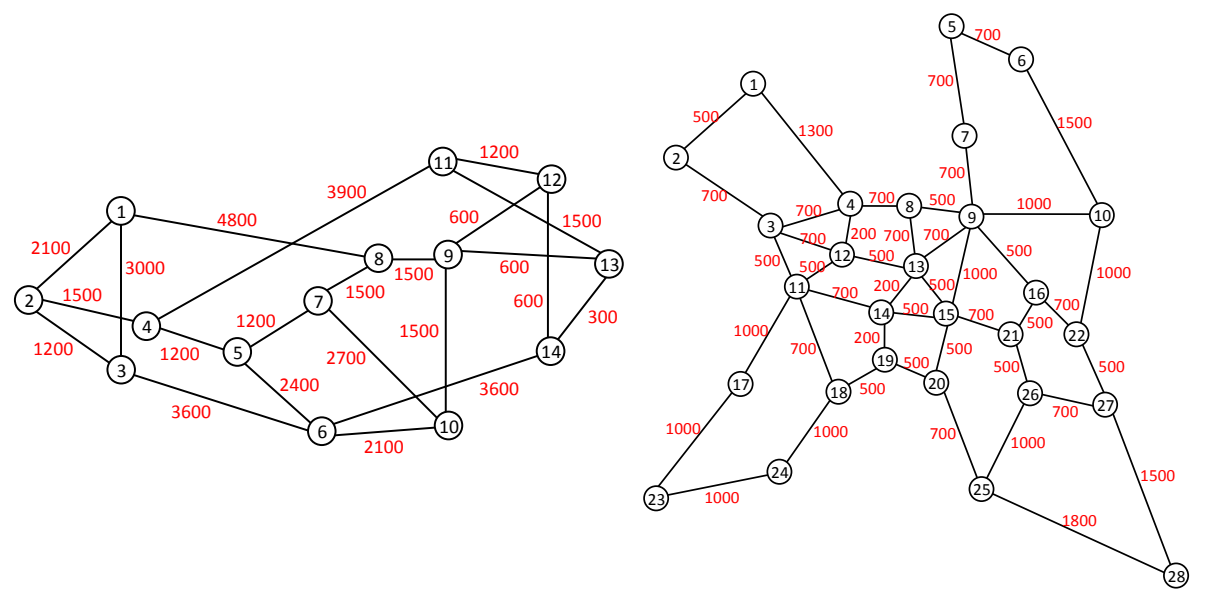

Figura 4. Topologias NSFNet e EON, respectivamente, com enlaces em km. 
Para a avaliação de desempenho, cada fibra possui sete núcleos, sendo cada núcleo dividido em 320 slots de frequência. Um slot de frequência possui largura de banda de 12,5 GHz. A banda de guarda possui largura de banda de um slot. Os ganhos dos amplificadores são ajustados para compensar as perdas dos dispositivos e da fibra. Outros parâmetros utilizados nas simulações estão listados na Tabela 1 [Beyranvand and Salehi 2013, Zhao et al. 2015, Lobato et al. 2019].

Tabela 1. Parâmetros de camada física utilizados nas simulações.

\begin{tabular}{c|c}
\hline Descrição & Valor \\
\hline Densidade espectral de potência do sinal & $-23 \mathrm{dBm} / \mathrm{GHz}$ \\
Atenuação da fibra $(\alpha)$ & $0,2 \mathrm{~dB} / \mathrm{km}$ \\
Parâmetro de dispersão da fibra $(D)$ & $16 \mathrm{ps}^{2} / \mathrm{km}$ \\
Coeficiente não linear da fibra $(\gamma)$ & $1,3(\mathrm{Wkm})^{-1}$ \\
Tamanho de um span $\left(L_{s}\right)$ & $80 \mathrm{~km}$ \\
Figura de ruído do amplificador $(N F)$ & $5 \mathrm{~dB}$ \\
Raio de curvatura $(R)$ & $0,01 \mathrm{~m}$ \\
Constante de propagação $(\beta)$ & $10^{7} 1 / \mathrm{m}$ \\
Distância entre núcleos $(\Lambda)$ & $4,5 \times 10^{-5} \mathrm{~m}$ \\
Coeficiente de acoplamento $(k)$ & $5,84 \times 10^{-3} \mathrm{~m}^{-1} \mathrm{ou} 1,2 \times 10^{-2} \mathrm{~m}^{-1}$ \\
\hline
\end{tabular}

São usados cinco formatos de modulação, sendo eles: BPSK, QPSK, 8-QAM, 16QAM e 32QAM. A distância máxima suportada por cada formato de modulação, juntamente com seus respectivos limiares de SNR por símbolo e de crosstalk são apresentados na Tabela 2 [Gong et al. 2013, Ives et al. 2015, Ehsani Moghaddam et al. 2019].

Tabela 2. Parâmetros de modulação e limiares de SNR e XT.

\begin{tabular}{c|c|c|c|c|c}
\hline & BPSK & QPSK & 8 -QAM & $16-Q A M$ & $32 Q A M$ \\
\hline Distância & $10.000 \mathrm{~km}$ & $5.000 \mathrm{~km}$ & $2.500 \mathrm{~km}$ & $1.250 \mathrm{~km}$ & $650 \mathrm{~km}$ \\
Limiar de SNR & $5,5 \mathrm{~dB}$ & $8,5 \mathrm{~dB}$ & $12,5 \mathrm{~dB}$ & $15,1 \mathrm{~dB}$ & $18,1 \mathrm{~dB}$ \\
Limiar de XT & $-14 \mathrm{~dB}$ & $-18,5 \mathrm{~dB}$ & $-21 \mathrm{~dB}$ & $-25 \mathrm{~dB}$ & $-27 \mathrm{~dB}$ \\
\hline
\end{tabular}

O primeiro cenário de avaliação considera um baixo nível de crosstalk, segundo [Lobato et al. 2019]. Para isso, o coeficiente de acoplamento $(k)$ é ajustado para 5,84 $\times$ $10^{-3} \mathrm{~m}^{-1}$ [Lobato et al. 2019]. A proposta é comparada com quatro algoritmos SCA: i) escolha aleatória de núcleo (Random Core) com alocação espectral first fit, nomeada RC-FF; ii) escolha aleatória de núcleo com alocação espectral random fit, nomeada de RC-RF; iii) escolha de núcleo através do algoritmo Core Prioritization [Fujii et al. 2014] e com alocação espectral first fit, nomeada CP-FF; e $i v$ ) escolha de núcleo através do algoritmo Core Prioritization e com alocação espectral random fit, nomeada de CP-RF. O algoritmo de Dijkstra é utilizado para escolha da rota em todos os algoritmos avaliados. A partir da definição de rota, o formato de modulação é selecionado considerando o alcance máximo permitido para a modulação. O resultado em termos de probabilidade de bloqueio de circuito é apresentado na Figura 5. A janela de observação (carga) é disposta para uma probabilidade de bloqueio de até 0,1 .

De acordo com a Figura 5, observa-se que o algoritmo ABNE obteve o melhor resultado em termos de probabilidade de bloqueio de circuito nas duas topologias. Os 


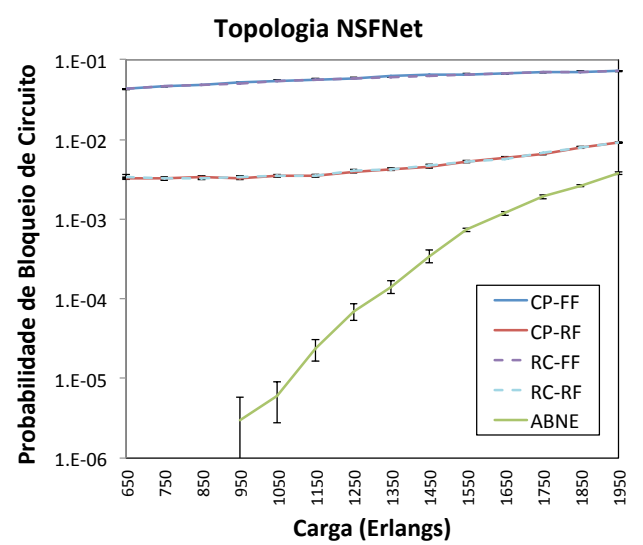

(a)

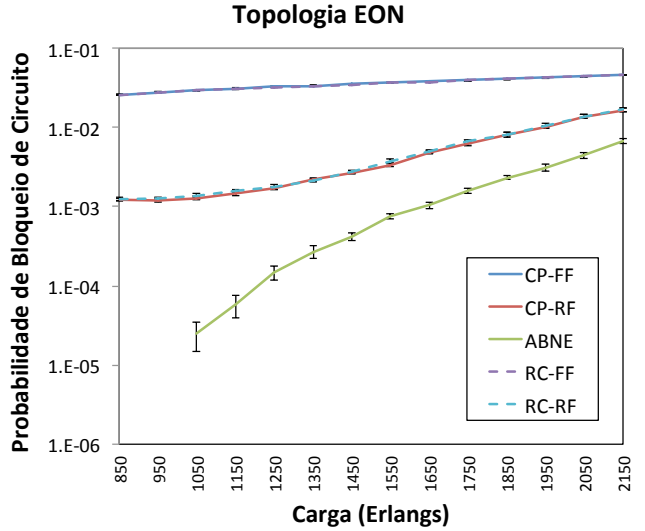

(b)

Figura 5. Probabilidade de bloqueio de circuito com baixo crosstalk. (a) Topologia NSFNet. (b) Topologia EON.

algoritmos CP-FF e RC-FF obtiveram resultados similares, tal qual os algoritmos CP-RF e RC-RF. Isso indica que, no cenário estudado, a estratégia de alocação espectral têm maior influência no bloqueio do que a estratégia de alocação de núcleo. Tal comportamento apresenta-se em outros trabalhos [Lobato et al. 2019]. Valores de probabilidade de bloqueio de banda são apresentados na Figura 6.

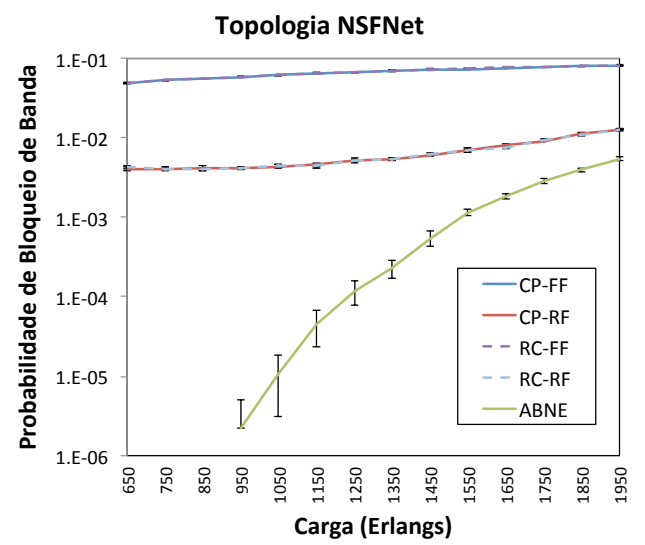

(a)

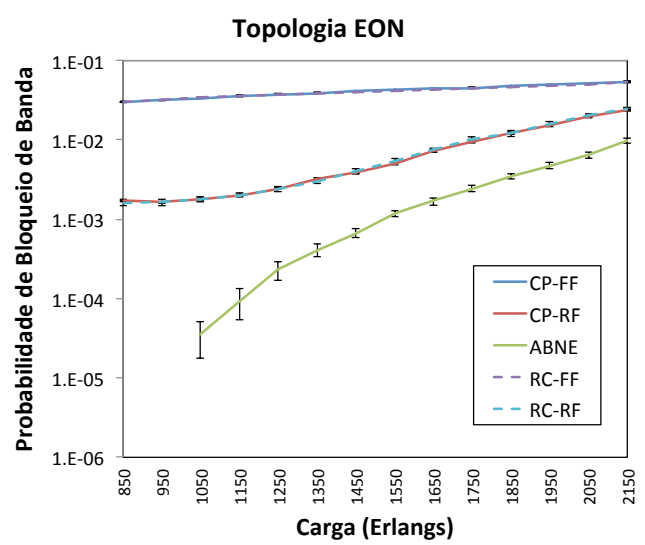

(b)

Figura 6. Probabilidade de bloqueio de banda com baixo crosstalk. (a) Topologia NSFNet. (b) Topologia EON.

A Figura 6 mostra um comportamento similar ao da Figura 5, onde o algoritmo ABNE obteve um melhor desempenho quando comparado aos concorrentes. Considerando todas as cargas analisadas na topologia NSFNet, o algoritmo ABNE reduziu a probabilidade de bloqueio de banda em pelo menos $93 \%$ em relação aos algoritmos CP-FF e RC-FF, e 57\% em relação aos algoritmos CP-RF e RC-RF. Na topologia EON, também considerando todas as cargas analisadas, o algoritmo ABNE reduziu a probabilidade de bloqueio de banda em pelo menos $82 \%$ em relação aos algoritmos CP-FF e RC-FF, e 59\% em relação aos algoritmos CP-RF e RC-RF.

O segundo cenário de avaliação considera um alto nível de crosstalk, segundo 
[Lobato et al. 2019]. Para isso, o coeficiente de acoplamento $(k)$ é ajustado para $1,2 \times$ $10^{-2} \mathrm{~m}^{-1}$ [Lobato et al. 2019]. Neste caso, os valores de probabilidade de bloqueio aumentaram consideravelmente em relação ao cenário anterior. A Figura 7 apresenta a probabilidade de bloqueio de banda dos algoritmos no cenário de alto crosstalk.

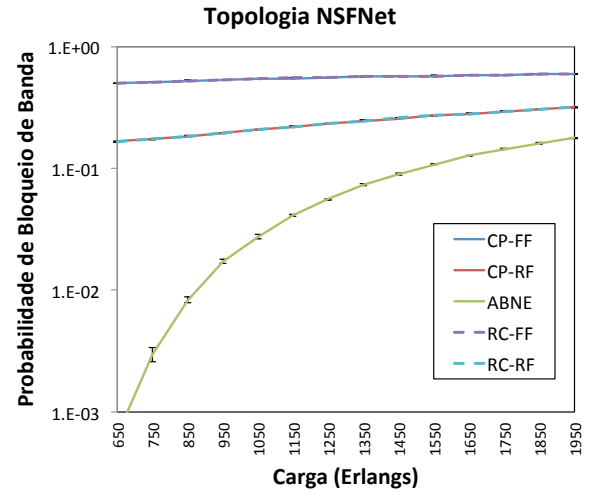

(a)

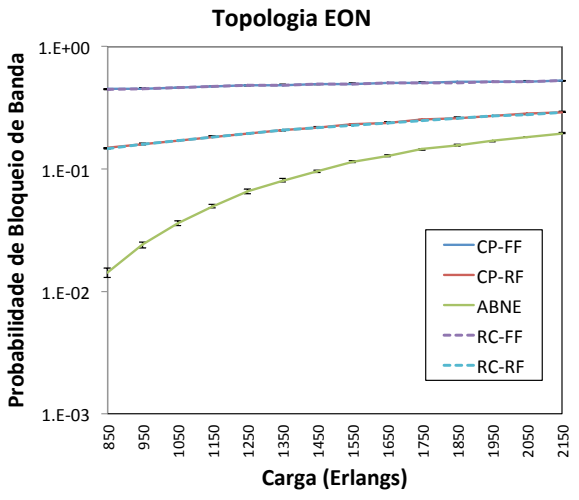

(b)

Figura 7. Probabilidade de bloqueio de banda (alto crosstalk). (a)NSFNet (b)EON.

De acordo com a Figura 7 observa-se que o ABNE obteve menores valores de probabilidade de bloqueio de banda quando comparado aos concorrentes em ambas as topologias. No entanto, o cenário de alto crosstalk trouxe um aumento considerável no nível de requisições bloqueadas. Considerando todas as cargas analisadas na topologia NSFNet, o algoritmo ABNE reduziu a probabilidade de bloqueio de banda em pelo menos $70 \%$ em relação aos algoritmos CP-FF e RC-FF, e 44\% em relação aos algoritmos CPRF e RC-RF. Na topologia EON, também considerando todas as cargas analisadas, o algoritmo $\mathrm{ABNE}$ reduziu a probabilidade de bloqueio de banda em pelo menos $63 \% \mathrm{em}$ relação aos algoritmos CP-FF e RC-FF, e 33\% em relação aos algoritmos CP-RF e RC-RF. A Figura 8 apresenta os componentes de bloqueio para os algoritmos avaliados. Buscando melhor visualização e considerando a similaridade entre os algoritmos CP-FF/RC-FF e $\mathrm{CP}-\mathrm{RF} / \mathrm{RC}-\mathrm{RF}$, apenas os resultados dos algoritmos ABNE, CP-FF e CP-RF são exibidos.

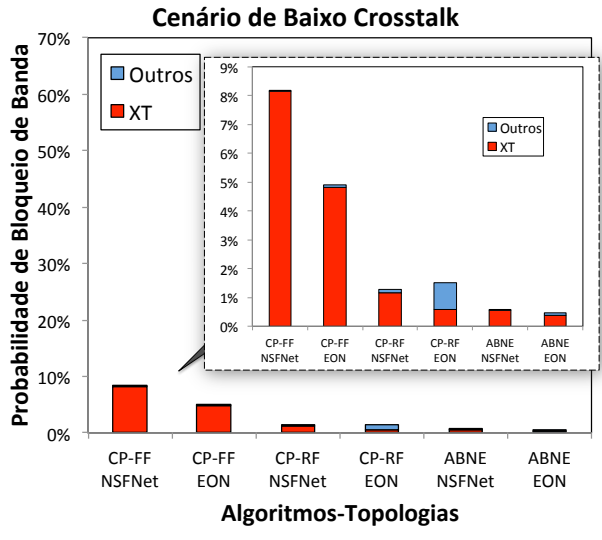

(a)

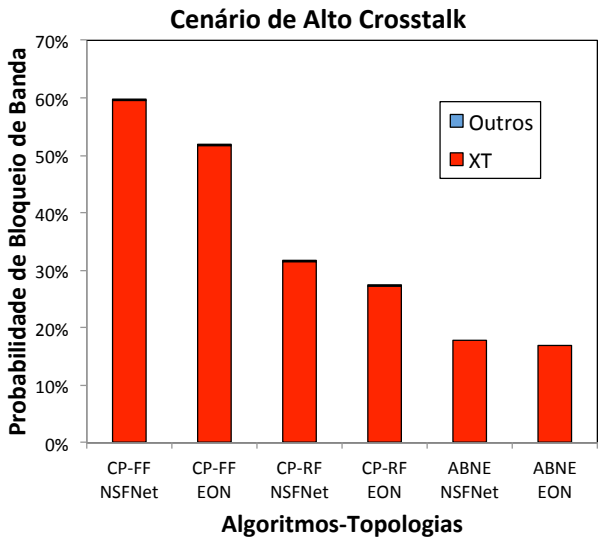

(b)

Figura 8. Componentes de bloqueio de banda nos cenário de: (a) baixo crosstalk. (b) alto crosstalk. 
A Figura 8 mostra a decomposição do bloqueio geral em suas causas específicas considerando as duas topologias, para o ponto de carga de 1950 Erlangs (maior ponto para a topologia NSFNet). Na Figura 8, o termo "Outros"compreende aos bloqueios causados por fragmentação, ausência de recursos, ausência de QoTN e ausência de QoTO. A Figura 8 (a) apresenta a probabilidade de bloqueio de banda no cenário de baixo crosstalk. Neste caso, o bloqueio causado por crosstalk influência a maior parte dos algoritmos, sendo o CP-FF o mais afetado pelo crosstalk. Esta sensibilidade ao crosstalk do algoritmo first fit dar-se a sua alocação espectral priorizar slots de índice menor, provocando uma concentração de circuitos nesta região e, consequentemente, alto crosstalk nestes circuitos. Evitar este tipo de situação é a proposta do $\mathrm{ABNE}$, que consegue valores menores de crosstalk devido ao seu balanceamento espectral. A Figura 8 (b) mostra a composição do bloqueio no cenário de alto crosstalk. Neste caso, o bloqueio causado por crosstalk é predominante em todos os algoritmos, sendo $99 \%$ da causa de seus bloqueios.

Além das probabilidades de bloqueio de circuito e de banda, os algoritmos são avaliados em função da sua eficiência energética. A eficiência energética é uma métrica que avalia a quantidade de bits transmitidos em relação a quantidade de energia consumida. O modelo de consumo de energia adotado [Vizcaíno et al. 2012, Ju et al. 2016] considera a contribuição dos transponders de largura de banda variável, comutadores ópticos de largura de banda variável e amplificadores EDFAs. O consumo de energia total da rede é calculado pela soma da energia total consumida pelos transponders, comutadores e EDFAs. A Figura 9 apresenta os resultados em termos de eficiência energética para os algoritmos ABNE, CP-FF e CP-RF no cenário de alto crosstalk.

Topologia NSFNet

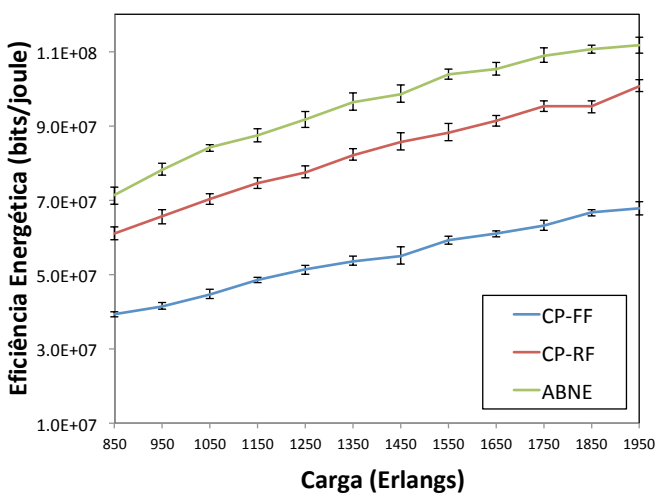

(a)

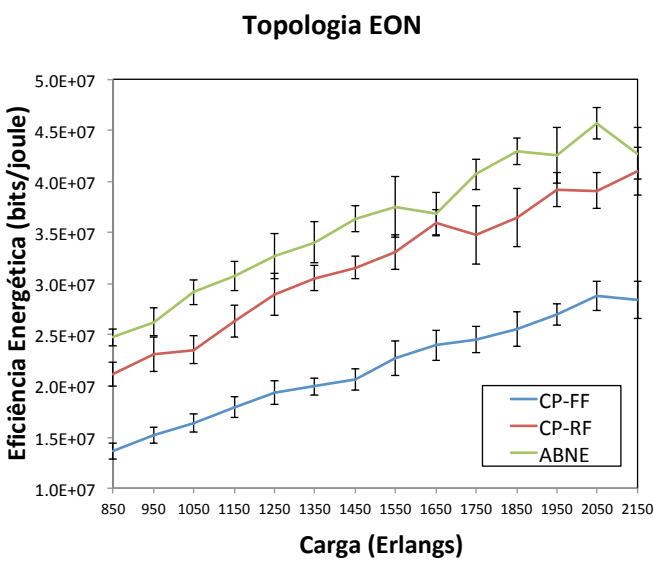

(b)

Figura 9. Eficiência energética (bits/joule) com alto crosstalk. (a) Topologia NSFNet. (b) Topologia EON.

Na topologia NSFNet, apresentada pela Figura 9 (a), o ABNE mostrou-se mais eficiente em relação aos demais algoritmos. Sendo em média $11 \%$ mais eficiente que o CP-RF e $61 \%$ em relação ao CP-FF. Na topologia EON (Figura 9 (b)), o ABNE mostrouse mais eficiente na maioria dos pontos de cargas, tendo ocorrido sobreposição de intervalos de confiança em cinco pontos de carga. Isso significa que nestes pontos, os algoritmos $\mathrm{ABNE}$ e CP-RF apresentam desempenho energético equivalente. 


\section{Conclusão}

Neste artigo é proposto o Algoritmo de Balanceamento de Núcleo e Espectro para Redes SDM (ABNE). O ABNE, ao alocar recursos de forma a mitigar o efeito do crosstalk, consegue obter bons resultados de acordo com as métricas de avaliação de desempenho usadas. A avaliação de desempenho foi conduzida em dois cenários: de baixo e alto crosstalk. Além disso, foram avaliadas duas topologias (NSFNet e EON).

O ABNE foi comparado com outros quatro algoritmos (RC-FF, RC-RF, CP-FF e CP-RF), sendo superior a todos em termos de probabilidade de bloqueio de circuito, probabilidade de bloqueio de banda e eficiência energética. O ABNE alcançou uma redução na probabilidade de bloqueio de banda de pelo menos $44 \%$ na topologia NSFNet e $33 \%$ na topologia EON. Técnicas de alocação espectral como o first fit ou last fit tendem a povoar sempre a mesma porção do espetro, aumentando assim o crosstalk entre os circuitos ópticos da rede. Entretanto, o uso destas técnicas de forma conjunta e sincronizada diminui a sobreposição espectral e, consequentemente, o crosstalk entre os circuitos.

Em trabalhos futuros, pretende-se expandir o estudo de avaliação de desempenho do algoritmo ABNE, comparando-o com outras propostas da literatura, usando novas métricas de avaliação de desempenho e novos cenários. Além disso, pretende-se criar um critério mais eficiente para a escolha do núcleo utilizado. Também pretende-se otimizar o uso do núcleo central, devido a sua sensibilidade e o impacto que ele causa nos demais núcleos. Por fim, pretende-se expandir a proposta para cenários de escolha de modulação adaptativa e que garanta sobrevivência na rede.

\section{Referências}

Beyranvand, H. and Salehi, J. (2013). A quality-of-transmission aware dynamic routing and spectrum assignment scheme for future elastic optical networks. Journal of Lightwave Technology, 31(18):3043-3054.

Chatterjee, B., Sarma, N., and Oki, E. (2015). Routing and spectrum allocation in elastic optical networks: A tutorial. IEEE Communications Surveys Tutorials, 17(3):1776 1800 .

Ehsani Moghaddam, E., Beyranvand, H., and Salehi, J. A. (2019). Crosstalk-aware resource allocation in survivable space-division-multiplexed elastic optical networks supporting hybrid dedicated and shared path protection. Journal of Lightwave Technology, pages $1-1$.

Fontinele, A., Santos, I., Neto, J. N., Campelo, D. R., and Soares, A. (2017). An efficient ia-rmlsa algorithm for transparent elastic optical networks. Computer Networks, 118:1 -14 .

Fujii, S., Hirota, Y., Tode, H., and Murakami, K. (2014). On-demand spectrum and core allocation for reducing crosstalk in multicore fibers in elastic optical networks. IEEE/OSA Journal of Optical Communications and Networking, 6(12):1059-1071.

Gong, L., Zhou, X., Liu, X., Zhao, W., Lu, W., and Zhu, Z. (2013). Efficient resource allocation for all-optical multicasting over spectrum-sliced elastic optical networks. IEEE/OSA Journal of Optical Communications and Networking, 5(8):836-847. 
Ives, D. J., Bayvel, P., and Savory, S. J. (2015). Routing, modulation, spectrum and launch power assignment to maximize the traffic throughput of a nonlinear optical mesh network. Photonic Network Communications, 29(3):244-256.

Jinno, M., Takara, H., Kozicki, B., Tsukishima, Y., Sone, Y., and Matsuoka, S. (2009). Spectrum-efficient and scalable elastic optical path network: architecture, benefits, and enabling technologies. IEEE Communications Magazine, 47(11):66-73.

Johannisson, P. and Agrell, E. (2014). Modeling of nonlinear signal distortion in fiberoptic networks. Journal of Lightwave Technology, 32(23):4544-4552.

Ju, M., Zhou, F., Xiao, S., and Zhu, Z. (2016). Power-efficient protection with directed $p$ cycles for asymmetric traffic in elastic optical networks. Journal of Lightwave Technology, 34(17):4053-4065.

Klinkowski, M. and Zalewski, G. (2019). Dynamic crosstalk-aware lightpath provisioning in spectrally-spatially flexible optical networks. IEEE/OSA Journal of Optical Communications and Networking, 11(5):213-225.

Lobato, F. R., Jacob, A., Rodrigues, J., Cartaxo, A. V., and Costa, J. (2019). Inter-core crosstalk aware greedy algorithm for spectrum and core assignment in space division multiplexed elastic optical networks. Optical Switching and Networking, 33:61 - 73.

Moura, P. M. and Fonseca, N. L. S. (2018). Routing , Core , Modulation Level , and Spectrum Assignment Based on Image Processing Algorithms. IEEE/OSA Journal of Optical Communications and Networking, 10(12):947-958.

Oliveira, H. M. N. S. and Fonseca, N. L. S. (2018). Protection, Routing, Modulation, Core, and Spectrum Allocation in SDM Elastic Optical Networks. IEEE Communications Letters, 22(9):1806-1809.

Oliveira, H. M. N. S. and Fonseca, N. L. S. (2019). Proteção e roteamento multicaminho em redes ópticas elásticas com multiplexação por divisão espacial. Simpósio Brasileiro de Redes de Computadores e Sistemas Distribuídos SBRC 2019, pages 487-500.

Rottondi, C., Martelli, P., Boffi, P., Barletta, L., and Tornatore, M. (2018). Modulation format, spectrum and core assignment in a multicore flexi-grid optical link. In Optical Fiber Communication Conference, page M3J.2. Optical Society of America.

Vizcaíno, J. L., Ye, Y., and Monroy, I. T. (2012). Energy efficiency analysis for flexiblegrid ofdm-based optical networks. Computer Networks, 56(10):2400 - 2419. Green communication networks.

Yan, L., Agrell, E., Wymeersch, H., Johannisson, P., Di Taranto, R., and Brandt-Pearce, M. (2015). Link-level resource allocation for flexible-grid nonlinear fiber-optic communication systems. IEEE Photonics Technology Letters, 27(12):1250-1253.

Zhao, J., Wymeersch, H., and Agrell, E. (2015). Nonlinear impairment-aware static resource allocation in elastic optical networks. Journal of Lightwave Technology, 33(22):4554-4564. 